\title{
Huge borderline mucinous tumour of ovary- A rare case report
}

\author{
Grace Willibell ${ }^{1}$, Deepa Shanmugham ${ }^{2}$, \\ ${ }^{1}$ Resident, ${ }^{2}$ Associate Professor, Dept. of Obstetrics \& Gynaecology, Aarupadai Veedu Medical College, \\ Kirumampakkam, Pondicherry, India \\ *Corresponding Author: \\ Email: drdeepabalamurugan@gmail.com
}

\begin{abstract}
Introduction: Huge borderline ovarian tumour are rare in modern medicine because of the awareness, earlier medical consultation and available ultrasound facility even in rural area. Here we report a case of huge ovarian mass proved to be borderline malignant.

Case Report: A 38 years old female presented with abdominal distension of rapid onset within 3 months. On examination, there was a cystic mass of $30 \times 20 \mathrm{~cm}$ arising from pelvis with variegated consistency. Ultrasound and CECT evaluation illustrated a well-defined cystic lesion with solid component arising from right ovary. With the working diagnosis of ovarian tumour, staging laparotomy was performed with total abdominal hysterectomy with bilateral salpingo oophorectomy. Histopathological examination revealed the tumour as borderline mucinous cystadenocarcinoma of ovary, without any local spread.
\end{abstract}

Keywords: Borderline ovarian tumour, Mucinous, Laparotomy.

\section{Introduction}

Epithelial tumor of ovary represents $65-75 \%$ of all ovarian tumors.

These tumors are divided into 3 different categories: benign, borderline and malignant.

The term "Borderline ovarian tumour" was introduced by the World Health Organization (WHO) and International Federation of Gynaecology and Obstetrics (FIGO) in $1971 .{ }^{1}$ The introduction of this entity represented a great advancement since it separated a group of ovarian tumour with a much better prognosis, stage-for-stage from conventional ovarian carcinoma. According to the WHO definition, borderline epithelial tumor lacks obvious invasion of the stroma and has mitotic activity and nuclear abnormalities, intermediate between clearly benign and unquestionably malignant tumor of similar cell. ${ }^{2}$ Huge borderline ovarian tumors are rare in modern medicine because of the awareness, earlier medical consultation and available ultrasound facility even in rural area. Here we report a case of huge ovarian mass proved to be borderline malignant.

\section{Case Report}

A 38 years old female P3L3, sterilized, presented with chief complaints of abdominal distension for 3 months which was insidious in onset with faster progression. She had loss of appetite and loss of weight. She had no history of vomiting and pain abdomen. She gave negative history for menstrual irregularities and leucorrhoea. Her bowel and bladder habits were normal. On examination, she was thin built, pale and her vitals were stable. Her abdominal examination revealed a cystic mass occupying almost all quadrants of abdomen of $30 \times 20 \mathrm{~cm}$ size, with variable consistency. Lower border of the mass was felt. Bimanual pelvic examination confirmed the findings and uterus was normal in size, mid position, mass felt separately, all fornices were full.

On evaluation, her complete blood count was within normal limits except for a low hemoglobin. Her investigations including liver and kidney function test, blood sugar, electrolytes and urine analysis were normal. USG showed a Cyst of size $30 \times 25 \mathrm{~cm}$ with heterogeneous solid component of $10 \times 12 \mathrm{~cm}$ size, occupying the abdomen and pelvis with peripheral vascularity. No free fluid in the abdomen. CT scan illustrated a well-defined cystic lesion of size $30 \times 25$ $\mathrm{cm}$ involving abdominopelvic region with large solid heterogeneous component along left lateral side of lesion arising from right ovary. Uterus and left ovary was normal. Pelvic and paraaortic nodes were not enlarged. Para colic and para rectal fat planes were normal. Normal enhancement of mesentery noted. Liver, spleen, both kidneys and ureters were normal. On intra venous pyelogram, both ureters were traced with functioning kidney. CA-125 value: 23.3 IU.

With the working diagnosis of ovarian cyst with the possibility of malignancy, she was planned for staging laparotomy. Abdomen opened in layers by vertical incision. There was no ascites. Peritoneal fluid samples were taken. Very huge cyst of $30 \times 25 \mathrm{~cm}$ arising from right ovary was removed intact. Careful inspection $\&$ palpation of intra-abdominal structures for implants was done. No evidence of peritoneal implants. Other ovary and uterus examined was found to be normal. Hysterectomy with left salpingo oophorectomy was performed. Omental biopsy was taken. Abdomen was closed in layers. On gross examination, ovarian cyst of $30 \times 25 \mathrm{~cm}$, smooth surface, $6 \mathrm{~kg}$ in weight (Fig. 1) Cut Section showed mucinous material in the cyst with loculations with the presence of solid area. Histopathological examination showed ovarian cyst wall lined by multilayered epithelium (more than four 
layers) and forming architectural complex pattern with branching and tufting. The lining epithelial cells were endocervical type and exhibits moderate degree of cytological atypia, foci of malignant nuclei, prominent nucleoli and increased atypical necrosis and destructive stromal invasion upto $3 \mathrm{~mm}$ (Fig. $2 \& 3$ ). The final impression was "Borderline mucinous tumor of ovaryendocervical type - intraepithelial carcinoma". Peritoneal fluid cytology and omental biopsy was negative for malignancy. Her postoperative period was uneventful. With the final diagnosis of borderline mucinous tumor of right ovary stage Ia, she was discharged on eighth post-operative day and was advised follow up. Patient is on regular follow up.

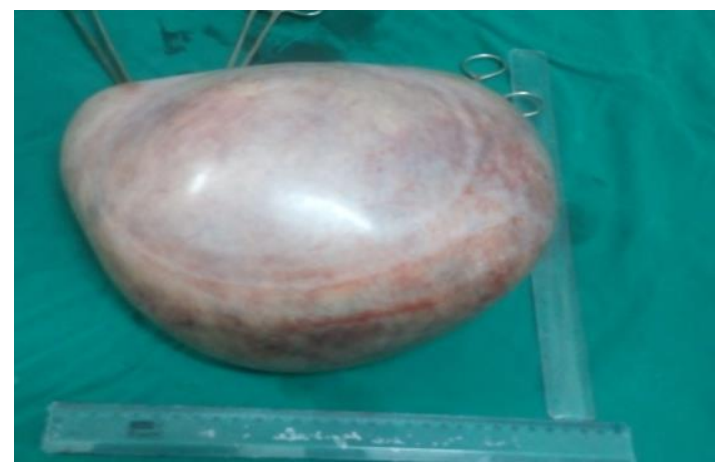

Fig. 1: Gross appearance of the tumour

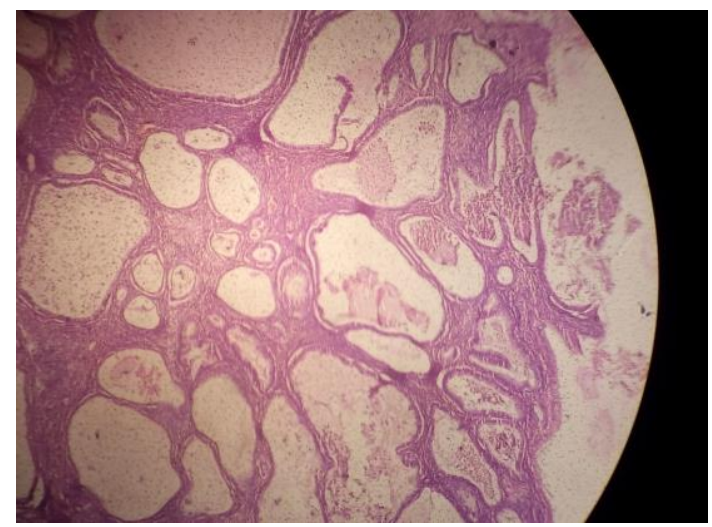

Fig. 2: Histopathological picture of the tumour

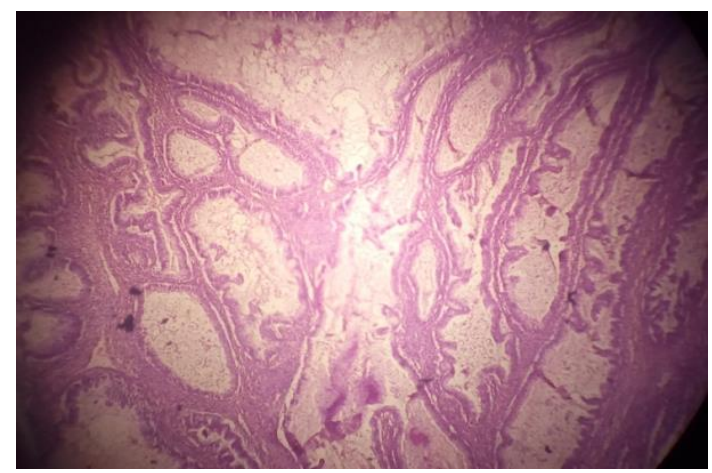

Fig. 3: Histopathological picture of the tumour

\section{Discussion}

Huge ovarian tumours are rare in modern medicine with the advent of diagnostic technology and public awareness. However in a developing country like India, women's health is still a great concern. Fortunately, our patient even though presented with a huge ovarian cyst, proved to be borderline tumor. Borderline ovarian tumors (BOT) are rare tumors with an incidence of 2.5/1, 00,000 women years. ${ }^{3}$ The great majority of BOTs are of serous and mucinous types. Mucinous BOTs are divided into endocervical like (mullerian) and intestinal subtypes. ${ }^{4}$ The intestinal type tumours are by far the most common and are usually large, unilateral with smooth capsule lined by tufted epithelium showing mild-moderate cytological atypia. ${ }^{5}$ The mucinous BOTs of endocervical type comprise $10-15 \%$ of mucinous BOTs and they resemble endocervical epithelium.

In a special pathological entity called mucinous borderline ovarian tumour with intra-epithelial carcinoma, as in our patient, the histological appearances are similar to the intestinal type borderline mucinous tumour but in addition, there will be foci of malignant nuclei often with highly stratified, solid or cribriform areas. ${ }^{6}$ Mucinous tumours may reach a huge size. Grossly, these tumours appear as rounded, ovoid or irregularly lobulated growth with smooth surface. Cut surface shows loculations. The content of the cyst is generally a clear viscid fluid, mucin.

Transvaginal ultrasound can detect the ovarian pathology with a high sensitivity (87-95\%) depending on the experience of the operator. Colour Doppler ultrasound which can illustrate the intracystic blood flow has a high sensitivity in differentiating malignancies from benign tumours. ${ }^{7}$ Computerised Tomography (CT) is required for adequate staging of the disease and evaluation of involvement of lymph nodes and other abdominal structures. Malignant and borderline ovarian tumours are formed of cells that contain high amounts of triglycerides in their membrane and, therefore show a characteristic spectrum in magnetic resonance imaging (MRI). MRI is a useful technique for predicting malignancy and for invasion and peritoneal implants.

Engelen et al studied the clinical significance of CA 125, CA 19-9 and CEA in borderline ovarian tumors. In patients with mucinous borderline ovarian tumors, the preoperative CA 19-9 level was more frequently elevated $(57 \%)$ than CA $125(15 \%)$ or CEA. ${ }^{8}$ Regarding the role of frozen section in the diagnosis of borderline ovarian tumours, a systemic review revealed that diagnostic accuracy rate for frozen section diagnosis is high for malignant \& benign tumor but diagnostic accuracy in borderline ovarian tumors remain low. ${ }^{9}$ The diagnosis of BOTs is difficult by frozen section, mainly due to their heterogeneity in appearance, especially in mucinous type.

The primary modality of management of borderline ovarian tumors remains surgery. A carefully done 
complete surgical staging is the ideal procedure. In early stage borderline ovarian tumors (stage I), ovarian cystectomy (either uni or bilateral), unilateral salpingooophorectomy and contralateral ovarian cystectomy have also been reported in the management with an increased risk of recurrence $(10-15 \%) .{ }^{10}$ This can be reserved for patients who need fertility sparing surgery. However our patient was near 40s and had completed her family, was treated with hysterectomy with bilateral salpingo oophorectomy to prevent any recurrence in the future.

In patients with advanced stage Borderline Ovarian Tumours, total abdominal hysterectomy with bilateral salpingo oophorectomy is the standard procedure together with omentectomy. Removal of all visible lesions to be attempted to give optimal clearance. Regarding lymph node sampling, in a study involving 42 patients with BOTs, where lymph node sampling was done in all of them, neither cases with mucinous tumor nor a case with early stage had nodal involvement. The authors concluded that routine lymphadenectomy is not justified in patients with early stage disease and in mucinous type. ${ }^{11}$

A Swedish study revealed that, in radically operated patients with low risk borderline tumors, the prognosis is very good and there is indication for adjuvant therapy. ${ }^{12}$ Chemotherapy is only indicated in the more aggressive borderline ovarian tumors eg. serous invasive implants and eventually pseudomxoma peritonei. Women with adequate staging and complete surgery, borderline ovarian tumors stage Ia and Ib, have a 5 year survival rate even upto $100 \%$ with a very low recurrence rate. ${ }^{13}$ Hence the prognosis of any early stage borderline ovarian tumor is better.

\section{References}

1. Tavassoli FA, Devilee P. World Health Organization classification of tumors. Pathology and Genetics of tumors of the breast and female genital organs. Lyon: IARC Press, 2003.

2. Prat J. Ovarian tumors of borderline malignancy (tumors of low potential): a critical appraisal. Adv Anat Pathol 1999;6:247- 274.

3. Sherman M E. Berman J, Birrer M J et al. Current challenges and opportunities for research on borderline ovarian tumors. Human Pathol 2004;35:961-7.

4. Siriaunkgul S, Robbins KM, McGowan L, Silverberg SG.Ovarian Mucinous tumours of low malignant potential: a clinicopathologic study of 54 tumours of intestinal and mullerian type.Int J Gynecol Pathol 1995; 14:198-208.

5. Hart WR. Mucinous tumors of the ovary: a review. Int $\mathbf{J}$ Gynecol Pathol 2005;24:4-25.

6. Ronnet BM, Kajdacsy-Balla A, Gilks CB et al. Mucinous Borderline ovarian tumors: points of general agreement and persistent controversies regarding nomenclature, diagnostic criteria, and behaviour. Hum Pathol 2004;35:949-960.

7. Zanetta G, Rota S, Lissoni A. Colour Doppler ultrasound in the preoperative assessment of adnexal masses. Acta Obstet Gynecol Scand 1994;73:637-41.
8. Engelen MJ, de Bruijn HW, Hollem H et al. Serum CA 125 , carcinoembryonic antigen and CA 19-9 as tumor markers in borderline ovarian tumors. Gynecol Oncol 2000;78:16-20.

9. Medeiros LR, Rosa DD, Edelweiss MI et al. Accuracy of frozen section analysis in the diagnosis of ovarian tumors: a systematic quantitative review. Int J Gynecol cancer 2005; 15; 192-202.

10. Gershenson D M. Clinical management potential tumours of low malignancy. Best prac Res Clin Obstet Gynecol 2002;16:513-27.

11. Camatte $\mathrm{S}$, Morice $\mathrm{P}$, Atallah D et al. Lymph node disorders and prognostic value of nodal involvement in patients treated for a borderline ovarian tumor: an analysis of a series of 42 lymphadenectomies. J Am coll surg 2002;195:332-338.

12. Hogberg T, Glimelius B, Nygren P. A Systemic overview of chemotherapy effects in ovarian cancer. Acta Oncol 2001;40:340-360.

13. Silverberg S G, Bell D A, Kurman R J et al. Borderline ovarian tumors: key points and workshop summary. Hum Pathol 2004;35:910-17. 